\title{
Evaluación de material didáctico de seguridad alimentaria con mujeres rurales del municipio de Pasto, Nariño (Colombia)
}

\author{
Yesica P. Ordoñez, Gloria C. Luna-Cabrera, Alejandra Narváez-Romo y Francisco Torres-Martínez \\ Universidad de Nariño, Facultad de Ciencias Agrícolas, Programa de Ingeniería Agroforestal. Calle 18 Carrera 50 \\ Ciudadela Universitaria Torobajo, Pasto, Nariño, Colombia. (correo-e: yekaordo159@gmail.com, grupopifil@gmail.com; \\ nrale.14@gmail.com; franjatm@hotmail.com)
}

Recibido Nov. 7, 2019; Aceptado Ene. 6, 2020; Versión final Ene. 30, 2020, Publicado Jun. 2020

\begin{abstract}
Resumen
El objetivo de esta investigación fue evaluar el material didáctico de seguridad alimentaria con participación de mujeres rurales en tres zonas del municipio de Pasto. Se aplicaron encuestas semi estructuradas para determinar factores de influencia en el aprendizaje durante los talleres de capacitación con el uso del material didáctico de seguridad alimentaria. Se utilizó un test en cuatro momentos con diseño experimental de dos factores: material didáctico y tiempo. Los factores de mayor influencia en el aprendizaje fueron: sociológico, psicológico y emocional; respecto al tiempo se evidenció que aún a largo plazo el aprendizaje sobre seguridad alimentaria es significativo; participativamente se planteó ajustes a los materiales para fortalecer el contenido incluyendo temas de soberanía alimentaria destacados en la región. Se concluye que el material didáctico empleado influye positivamente en la formación de mujeres rurales considerándolo innovador al generar motivación desde principios de respeto cultural, rescate de valores y tradiciones locales.
\end{abstract}

Palabras clave: aprendizaje de adultos; material didáctico; seguridad alimentaria; soberanía alimentaria

\section{Assessment of food security didactic material for rural women from the municipality of Pasto, Nariño (Colombia)}

\begin{abstract}
The objective of this research was to evaluate didactic material for food security with the participation of rural women from three areas in the municipality of Pasto. Semi-structured surveys were applied to determine factors that influence learning during training workshops by using didactic material on food security. This was tested at four different times using a two-factor (didactic material and time) experimental design. The most influential factors observed in the learning process were sociological, psychological and emotional. In terms of time, learning about food security was significant in the long term. Participatory adjustments were made to the materials to strengthen food sovereignty issues important for the region. It was concluded that the didactic material had a positive influence on the training of rural women. The didactic material was considered innovative because it generated motivation for cultural respect principles and because it rescued local values and traditions.
\end{abstract}

Keywords: adult learning; didactic material; food security; food sovereignty 


\section{INTRODUCCIÓN}

La presencia de Tecnologías de la Información y la Comunicación (TIC), ha crecido de forma significativa y se han convertido en catalizadoras de cambio, capaces de provocar modificaciones en los procesos de enseñanza y aprendizaje. Las TIC agrupan elementos y técnicas usadas en el tratamiento y transmisión de información; son herramientas que pueden ser aprovechadas para la construcción de material didáctico, facilitando el aprendizaje y el desarrollo de habilidades, al requerir que el participante movilice su pensamiento crítico y analítico, mientras interactúa con ellas (Acosta, 2016). De esta manera se han convertido en una herramienta útil para la andragogía y en procesos de formación impartidos por extensionistas innovadores.

La andragogía es la disciplina que se ocupa de la educación y aprendizaje del adulto, quien busca el conocimiento para su aplicación inmediata, lo cual le permite obtener frutos en el menor tiempo. Consiste en aprender a conocer, aprender a aprender, aprender a hacer y aprender a ser, así como sus características, basado en el conocimiento útil, la experiencia y el funcionamiento psicológico del adulto en el entorno en que éste se desenvuelve y sus relaciones sociales con el mundo circundante y sus intereses multidimensionales, con el fin de orientar el aprendizaje a la elaboración de productos, al trabajo interdisciplinario y a la posibilidad de generalizar (Moore y Shemberger, 2019). En este mismo contexto, la enseñanza para adultos implica una metodología didáctica diferenciada, es importante entender que el adulto tiene unas características de aprendizaje y necesita de estrategias adecuadas para su formación, de igual manera el uso pertinente de las TIC, implica lograr aprendizaje placentero, partiendo de identificar aquellos aprendizajes individuales y colectivos que generan este éxtasis (Tipantuña, 2019).

En este sentido, es importante evaluar de manera objetiva los materiales didácticos para garantizar que los adultos pueden aprender a través ellos de forma autónoma. López y Malagón, (2017) mencionan que es sustancial que los extensionistas usen materiales que sean pertinentes y significativos para quienes va dirigido, de igual forma que sus contenidos sean de calidad. Las tendencias nacionales e internacionales sobre la evaluación de los sistemas educativos indican su consolidación y expansión en todos los ámbitos del proceso de enseñanza-aprendizaje. La evaluación de un material toma más sentido cuando se sabe si lo que se ha diseñado es válido y útil en relación con su finalidad. Es importante realizar una evaluación inicial; una evaluación continua durante el proceso, y una evaluación final, que permitirán hacer los cambios necesarios y sobre todo alcanzar satisfacción, si han contribuido a la innovación educativa en la búsqueda del mejoramiento de los procesos de enseñanza y aprendizaje (Carr et al., 2018).

En Nariño, se han evaluado en diferentes zonas, materiales didácticos, como es el "software educativo mundo agroforestal" con jóvenes rurales (Narváez-Romo et al., 2017) y el "material didáctico Agroforesteria" con agricultores (Yaqueno, et al., 2018), los cuales han facilitado el aprendizaje significativo, que se evidencia durante el uso de éstos materiales que generan una mayor compresión y estimulan la apropiación del conocimiento en comunidades rurales. Sin embargo, a pesar de que las TIC son importantes, su evaluación en cuanto a la injerencia de éstas en la formación aún es incipiente (Yaqueno et al., 2018), en este sentido, el grupo de investigación PIFIL de la Universidad de Nariño, en Colombia ha diseñado de manera interdisciplinaria y contextualizada materiales didácticos como el de seguridad alimentaria para facilitar procesos de formación de comunidades rurales mediante cinco juegos (Rol familiar; Planifica tu producción; Mercado familiar; Concéntrese en su salud y El sapo alimenticio) que orientan el abordaje de distintas temáticas enmarcadas en cinco módulos de capacitación (la familia y su compromiso con la producción, disponibilidad y diversidad de alimentos, importancia de la huerta en la economía familiar, promoción de la salud y alimentación saludable).

Considerando, que en el departamento de Nariño las familias con producción en minifundio presentan limitado nivel de formación respecto a seguridad alimentaria evidenciándose falencias en cuanto al reconocimiento de la importancia de una adecuada alimentación, y que además no se encuentran establecidos aquellos factores que limitan o impulsan el aprendizaje, es evidente la necesidad de un análisis participativo con mujeres rurales que en su mayoría son quienes asumen el rol de la preparación de alimentos para sus familias y que con frecuencia también se encargan de las huertas; por ello, es valioso su aporte desde la evaluación en cada uno de los módulos de capacitación con el uso de los materiales didácticos, contribuyendo con éstas herramientas innovadoras de formación, pertinentes a la dinámica cultural, contextualizadas y de mayor impacto para promover hábitos alimenticios saludables, en el marco de una agricultura familiar.

El objetivo de ésta investigación fue evaluar el material didáctico de seguridad alimentaria con la participación de mujeres rurales en tres corregimientos del municipio de Pasto, Nariño; identificando factores que influyen en su aprendizaje, analizando en el tiempo la comprensión de las temáticas de seguridad alimentaria impartidas en capacitaciones durante las cuales se empleó los juegos que integran dicho material, planteando a su vez ajustes resultado de los aportes compartidos por los participantes en el contexto local. 


\section{METODOLOGÍA}

La investigación se realizó en el municipio de Pasto, en los corregimientos de Obonuco, Genoy y La Laguna, situados a 2400, 2400 y 2800 metros sobre el nivel del mar respectivamente, con una temperatura promedio entre 8 a $12{ }^{\circ} \mathrm{C}$, con una distancia de la ciudad de Pasto de 5, 10 y 12 kilómetros; zona caracterizada por su vocación agrícola y pecuaria (Cadena, 2013). Se trabajó con 40 mujeres rurales pertenecientes a la Asociación agropecuaria ambiental y popular del Galeras-ASOAGROGALERAS, las cuales fueron seleccionadas de acuerdo a los siguientes criterios adaptados según los postulados propuestos por Chacón (2012): voluntad de participación, disponibilidad de tiempo, edad mayor de 18 años, permanencia durante todo el desarrollo del proyecto y disposición para la aplicabilidad de los conocimientos adquiridos.

Mediante investigación de tipo mixto con enfoque descriptivo, se llevó a cabo una revisión documental para disponer de mayor conocimiento de la zona de estudio y plantear una estrategia pertinente para adelantar la socialización del proyecto en la cual se seleccionaron las mujeres participantes considerando los criterios descritos anteriormente. Para la identificación de factores de aprendizaje se aplicaron 18 encuestas semiestructuradas diseñadas y adaptadas con base a la metodología de Dunn et al (1979), quienes determinan que las variables psicológico, emocional, perceptivo, fisiológico, ambiental y sociológico son modalidades de percepción de la información que influyen en el proceso de enseñanza y aprendizaje. En la Tabla 1 se muestra una descripción de las variables de factores de aprendizaje, cuyos resultados se analizaron con el promedio de la suma de puntajes obtenidos en cada pregunta, donde se manejó una escala de 0 de no influencia y 1 de influencia en el aprendizaje.

Tabla 1: Descripción de variables de factores de aprendizaje

\begin{tabular}{|l|l|l|}
\hline Encuesta & Variables & Descripción \\
\hline \multirow{1}{*}{$\begin{array}{l}\text { Factores de } \\
\text { aprendizaje }\end{array}$} & Sociológico & $\begin{array}{l}\text { Trabajo individual o colectivo y su influencia en la construcción de } \\
\text { conocimiento de acuerdo al contexto. }\end{array}$ \\
\cline { 2 - 3 } & Psicológico & Análisis y reflexión de la realidad y su aplicación práctica. \\
\cline { 2 - 3 } & Emocional & Motivación y dedicación a las actividades para el aprendizaje. \\
\cline { 2 - 3 } & Perceptivo & $\begin{array}{l}\text { Uso de los sentidos (auditivo, visual, kinestésico, verbal) para el } \\
\text { conocimiento. }\end{array}$ \\
\cline { 2 - 3 } & Ambiental & $\begin{array}{l}\text { Ambientes de trabajo que generan bienestar y satisfacción en los } \\
\text { participantes. }\end{array}$ \\
\cline { 2 - 3 } & Fisiológico & $\begin{array}{l}\text { Influencia del bienestar integral y su relación con la alimentación y } \\
\text { jornadas de trabajo. }\end{array}$ \\
\hline
\end{tabular}

A partir de lo anterior, y considerando cómo aprenden los adultos, se planificó el proceso de formación en cinco módulos de capacitación mediante el desarrollo de talleres, empleando el material didáctico de seguridad alimentaria que comprende el uso de los cinco juegos (rol familiar, planifica tu producción, mercado familiar, concéntrese en su salud y el sapo alimenticio) que facilitan la comprensión de los contenidos. Durante la realización de talleres, se aplicó un test de aprendizaje elaborado por un equipo interdisciplinario quienes abordaron integralmente los aspectos fundamentales para cada temática de los módulos de capacitación que comprende el material didáctico de seguridad alimentaria, el cual fue validado y aplicado a diez participantes en diferentes momentos durante el desarrollo de los talleres de capacitación teniendo en cuenta lo planteado por Aguilar (2011) con un diseño experimental de dos factores de análisis: material didáctico y tiempo.

El factor tiempo, se analizó a través de la determinación de variables y diseño de test como se muestra en la Tabla 2, que permitieron identificar conocimientos previos (Ev1) y generales aplicables en el corto (Ev2), mediano (Ev3) y largo plazo (Ev4), comprendidos en cuatro momentos: antes de realizar los talleres de capacitación (Ev1), una vez terminados los talleres de capacitación (Ev2), tres meses después de la última capacitación (Ev3) y seis meses después de la última capacitación (Ev4); el factor de evaluación del material didáctico, se realizó mediante el modelo ajustado de evaluación Stufflebeam (Mora, 2004) que considera cuatro etapas conocidas como CIPP: a) Contexto, b) Input (entrada), c) Proceso y d) Producto. Esta evaluación comprendió la revisión de fuentes secundarias como insumo esencial en la gestión del contenido en procesos de capacitación, la codificación, categorización y significancia de la información por parte de los participantes según lo propuesto por Aguilar (2011) mediante preguntas que estimen la percepción, comprensión y adoptabilidad. Para evaluar los resultados de cada participante, se empleó la escala de valoración desempeño bajo $(0,0-2,9)$ indicando la no aprobación, desempeño básico $(3,1-4,0)$, desempeño alto $(4,1-4,5)$ y desempeño superior $(4,6-5,0)$ indicando la aprobación de los contenidos en diferentes niveles de conocimiento. El análisis estadístico de los resultados se realizó por medio del programa SPSS 22.3, validando los datos con modelo lineal general con medidas repetidas para evaluar los conocimientos intrasujetos (tiempo) e intersujetos (lugar). 
Tabla 2: Variables de evaluación

\begin{tabular}{ll}
\hline Evaluación & Variables \\
\hline Test & Características de la seguridad alimentaria \\
& Personas que aportan a la seguridad alimentaria \\
& Beneficios del huerto familiar \\
& Clasificación de los alimentos \\
& Alimentos de la canasta familiar \\
& Alternativas a la seguridad alimentaria \\
& Producción permanente \\
\hline
\end{tabular}

Según Falcao et al. (2016), para la evaluación de este tipo de recursos, los criterios de evaluación se basan en sus propiedades físicas como la calidad de los materiales, su funcionalidad y la ergonomía adecuada al usuario. En la Tabla 3 se puede apreciar las variables de calidad y pertinencia de cada uno de los juegos del material didáctico de seguridad alimentaria que se aplicaron mediante una encuesta semiestructurada, para la toma de decisiones frente a los ajustes de fondo y de forma del material empleado en las capacitaciones. El resultado final alcanzado fue socializado con las mujeres participantes quienes evidenciaron sus contribuciones en los juegos rediseñados.

Tabla 3: Calidad y pertinencia de los juegos del material didáctico de seguridad alimentaria

\begin{tabular}{ll}
\hline Encuesta & Variables \\
\hline Calidad y pertinencia de los $\begin{array}{l}\text { juegos del material } \\
\text { didáctico de seguridad } \\
\text { alimentaria }\end{array}$ & Utilidad \\
\cline { 2 - 2 } & Facilidad de uso \\
\cline { 2 - 2 } & Claridad en instrucciones \\
\cline { 2 - 2 } Aplicabilidad de los contenidos en la práctica \\
\hline & Diseño gráfico \\
\hline & Tamaño y forma \\
\cline { 2 - 2 } & Resistencia y durabilidad \\
\hline & Aceptación \\
\hline & Pertinencia de los contenidos \\
\hline
\end{tabular}

\section{RESULTADOS Y DISCUSIÓN}

La población participante en esta investigación se encuentra en un rango de edad entre 40 a 50 años, en su mayor porcentaje con escolaridad de primaria incompleta en un $96 \%$ de las mujeres, caracterizadas por habitar en predios de minifundio con promedio de 1.5 hectáreas, dedicadas principalmente a la siembra de maíz (Zea mays L.), cebolla (Allium fistulosum L.) y papa (Solanum tuberosum L.); sus estructuras de organización comunitaria aún son insipientes y la asistencia técnica que reciben es limitada, al igual que los procesos de orientación en seguridad alimentaria. Mediante el análisis del modelo lineal general con medidas repetidas en relación a cómo son sus procesos de aprendizaje para mejorar su condición económica principal, que es la producción agrícola, se obtuvieron los siguientes resultados:

\section{Factores de aprendizaje}

Los índices de cada factor, indican que los de mayor influencia en el aprendizaje de mujeres rurales en los tres corregimientos de estudio son aquellos con mayor media, como se muestra en la Figura 1, el primer factor es el sociológico (16) =0,7039; en segundo lugar, el factor psicológico (I2) =0,7006 y por último el factor emocional $(I 5)=0,6878$; los factores perceptivo $(I 1=0,6367)$, ambiental $(I 3=0,5994)$ y fisiológico $(I 4=0,5561)$ presentaron la menor influencia.

Con respecto al factor sociológico, se encontró que a las mujeres rurales el trabajo colectivo les genera mayor aprendizaje; al respecto, Johnson y Johnson (1991), mencionan que el trabajo en grupos pequeños genera el máximo aprendizaje propio y entre sí, ya que el diálogo contribuye a la adquisición de conocimiento. Se ha demostrado que la actividad cooperativa y colaborativa resulta muy fructífera para solucionar diversas tareas y produce avances cognitivos significativos (Vasilaky e Islam, 2018); además cuando se trabaja en cooperación se adquieren estrategias de aprendizaje más eficaces y se resuelven problemas con un mayor éxito (De Backer et al, 2015). 


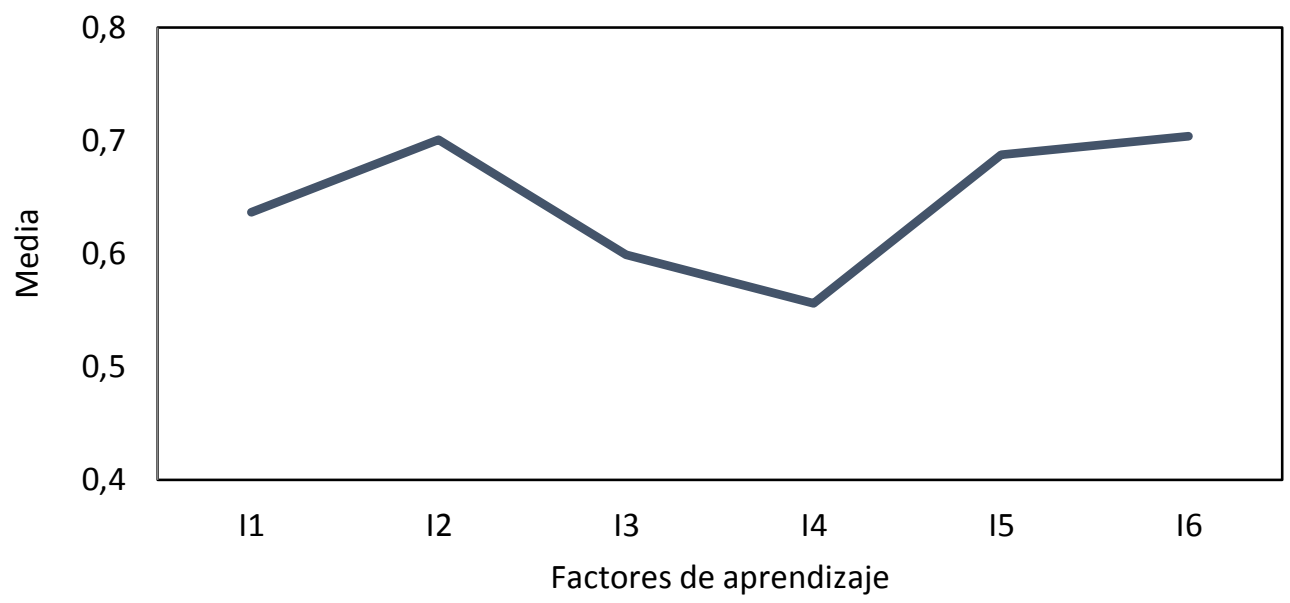

Fig. 1: Medias estimadas para factores de aprendizaje

Según Lu Gram et al (2019), el aprendizaje está en dependencia del intercambio de información, socialmente estructurado, entre cada uno de los integrantes del grupo y en el cual cada uno es responsable de su propio aprendizaje y se le motiva para aumentar el de los demás. Sin duda, cualquier actividad de aprendizaje cooperativo constituye una experiencia y reporta numerosos beneficios para cada individuo, este aprende a trabajar realmente en equipo y adquiere habilidades sociales.

El trabajar con grupos pequeños genera mayor comprensión sobre la realidad; se fortalece las relaciones entre los participantes al ayudarse mutuamente para lograr un mismo objetivo; genera la participación activa, independiente y creativa durante el proceso de enseñanza aprendizaje; aumenta la capacidad para definir, reconocer y solucionar problemas; los integrantes aprenden unos de otros, estimulando así el pensamiento crítico lo cual involucra activamente a los proceso de aprendizaje (Sánchez et al, 2018), además el trabajo participativo ayuda a que un individuo aprenda más de lo que aprendería por sí solo, fruto de la interacción de los integrantes de un equipo, quienes saben diferenciar y contrastar sus puntos de vista, de tal manera, que llegan a generar un proceso de construcción de conocimiento.

En relación al factor psicológico, donde predomina el aprendizaje por medio de análisis y reflexión de la realidad, De Backer et al (2015) menciona que el aprendizaje se adquiere, a través de un ciclo de experiencia, reflexión y acción donde el reflexionar sobre experiencias conlleva a una mejora en la comprensión; además señala que las situaciones de la vida real generan aprendizaje, ya que se adquiere conocimientos, habilidades y actitudes, por tanto, compartir estos espacios es de mutuo beneficio. De igual manera Caniglia et al (2016), exponen que los adultos aprenden mejor mediante experiencias cuando se les da la oportunidad de reflexionar sobre éstas y ejecutar una acción apropiada; por ello se necesitan métodos de capacitación interactivos, es decir, una comunicación abierta entre el facilitador y los participantes.

Por otra parte, Acosta (2016) menciona que la educación de personas adultas, la experiencia juega un papel muy importante, ya que con base en esta se fundamentan los nuevos aprendizajes o barreras que esta interponga para anclar o no su aprendizaje previo con el deseado. La riqueza del ser adulto radica en su experiencia, en lo que sabe, lo que ha vivido, lo que ha realizado, lo que piensa y siente. Esta, es heterogénea, y enriquecerla para mejorarla, adaptarse y poder resolver de manera satisfactoria las problemáticas a las que se enfrenta, es una de las principales motivaciones para diseñar e implementar procesos educativos permanentes (Leberman et al., 2016).

En cuanto al factor emocional, es de resaltar que la motivación y persistencia son importantes para el aprendizaje; según Alario-Hoyos et al (2017), estos son fundamentales en el proceso de aprendizaje, en tanto que los impulsa a la acción, a la vez que los orienta y los compromete, donde cada individuo construye su aprendizaje según sus conocimientos, esquema, saberes, experiencias previas, y su contexto en la interacción con los otros. De igual manera, Meij et al (2015) considera la motivación hacia el aprendizaje como un proceso determinado por las necesidades e intereses del individuo, que originan la voluntad de aprender, lo que incide positivamente en la disposición favorable ante el conocimiento. Para De Souza et al (2016), la motivación influye de manera significativa en las dimensiones: cognitiva, asociativa y autonomía del aprendizaje. Además, constituye un requisito fundamental que desencadena el aprendizaje. El deseo de aprender, las necesidades individuales y las perspectivas futuras impulsan al individuo a comprender más rápida y efectivamente. Suele ser un proceso individual y es sentida por cada ser humano de acuerdo a su historia personal. De Souza et al (2016) infiere que un facilitador puede provocar o maximizar tal necesidad 
en su alumno, por medio de estrategias pedagógicas adecuadas. Rajaee (2015), en su investigación señala que los materiales didácticos generan motivación e interés por aprender, ya que ayudan a captar la atención y la apropiación de conocimientos, brindando una experiencia significativa, que posibilita profundización de las temáticas tratadas.

\section{Análisis de la prueba intrasujetos}

Con respecto al factor tiempo, en la Tabla 4 se presenta por medio del modelo lineal general con medidas repetidas, los estadísticos (Traza de Pillai, Lambad de Wilks, Traza de Holleting y Raíz Mayor de Roy) mostraron diferencias estadísticamente significativas en los grupos $(P=0,000<0,05)$ y una $F$ invariada de 103,17 significativa $(p=0,00>0,005)$ lo que indica que el aprendizaje se mantuvo a través del tiempo al emplearse el uso del material didáctico, resultados similares encontrados en investigaciones de Narváez et al (2017) y Yaqueno et al (2018).

Tabla 4: Pruebas multivariantes del test general

\begin{tabular}{lllcccc}
\hline Efecto & \multicolumn{1}{c}{ Estadístico } & Valor & F & $\begin{array}{c}\text { Gl de } \\
\text { hipótesis }\end{array}$ & $\begin{array}{c}\text { gl de } \\
\text { error }\end{array}$ & Sig. \\
\hline Evaluaciones & Traza de Pillai &, 875 & $58,071^{\mathrm{b}}$ & 3,000 & 25,000 &, 000 \\
\cline { 2 - 7 } & Lambda de Wilks &, 125 & $58,071^{\mathrm{b}}$ & 3,000 & 25,000 &, 000 \\
\cline { 2 - 7 } & Traza de Hotelling & 6,969 & $58,071^{\mathrm{b}}$ & 3,000 & 25,000 &, 000 \\
\cline { 2 - 7 } & Raíz mayor de Roy & 6,969 & $58,071^{\mathrm{b}}$ & 3,000 & 25,000 &, 000 \\
\hline $\begin{array}{l}\text { Evaluaciones } \\
\text { Lugar }\end{array}$ & Traza de Pillai &, 446 & 2,488 & 6,000 & 52,000 &, 034 \\
\cline { 2 - 7 } & Lambda de Wilks &, 592 & $2,502^{\mathrm{b}}$ & 6,000 & 50,000 &, 034 \\
\cline { 2 - 7 } & Traza de Hotelling &, 627 & 2,508 & 6,000 & 48,000 &, 034 \\
\cline { 2 - 7 } & Raíz mayor de Roy &, 500 & $4,333^{\mathrm{c}}$ & 3,000 & 26,000 &, 013 \\
\hline
\end{tabular}

Silva et al (2016), afirman que la aplicación de materiales didácticos, junto con un buen método de enseñanzaaprendizaje, facilita la adquisición del conocimiento a través de una manera participativa y lúdica, brindando una experiencia significativa. Por otra parte, Sánchez et al (2018), plantea que los materiales no solo sirven para trasmitir información, sino también funciona como mediadores entre la realidad y los adultos, y mediante sus sistemas simbólicos desarrollan habilidades cognitivas, además que permiten motivar, facilitan la adquisición de nuevos conocimientos y apoyan a la evaluación y el reforzamiento del aprendizaje. Actualmente el uso de las TIC ha ayudado en el proceso de aprendizaje debido a que mejoran las oportunidades para la reflexión, el análisis, la resolución de problemas, el pensamiento crítico, así como el desarrollo de habilidades de comunicación, colaboración, aprendizaje independiente y de trabajo en grupo (Muñoz y Tejedor, 2017).

Teniendo en cuenta la importancia de los materiales didácticos, se puede inferir que el uso del material didáctico de seguridad alimentaria, el cual consta de cinco juegos, influyó positivamente en el proceso de aprendizaje de mujeres rurales, ya que es más factible recordar lo que se practica y genera emociones que marcan la diferencia y más aún cuando se crea un ambiente lúdico que genera confianza, integración y participación que conlleva a una apropiación del conocimiento. Zagarra et al (2016), mencionan que la educación por medio de la lúdica integra en su esencia una concepción teórica profunda y una concepción práctica actuante y concreta. Sus objetivos son la estimulación de las reacciones cognitivas, afectivas, verbales, psicomotoras, sociales, la mediación socializadora del conocimiento y la provocación de una reacción activa, crítica y creativa, lo que denominamos formación integral del ser humano (Nowak, 2018). Otros autores, como Walsh et al (2017), muestran la lúdica como un instrumento efectivo para la enseñanza, la cual unifica los contextos sociales, exaltando la cultura y creando nuevas formas de relación humana, unificando la didáctica y la pedagogía para facilitar los procesos de enseñanza y aprendizaje.

Por otra parte, Beltrán (2017) menciona que en el juego se pueden crear situaciones de máximo valor educativo y cognitivo que permiten experimentar, investigar, resolver problemas, descubrir y reflexionar. Las implicaciones de tipo emocional, el carácter lúdico, el desbloqueo emocional, la desinhibición, son fuentes de motivación que proporcionan una forma distinta a la tradicional de acercarse al aprendizaje. De igual forma, Chacón (2012) indica que los juegos requieren de la comunicación generando la activación de mecanismos de aprendizaje, además de desarrollar sus propias estrategias. Con el juego, los docentes dejan ser el centro de la clase, para pasar a ser facilitadores-conductores del proceso de enseñanza- aprendizaje, además de potenciar con su uso el trabajo en pequeños grupos o parejas. Yaqueno et al (2018) plantea que el juego 
ofrece numerosas ventajas en el proceso de enseñanza-aprendizaje como aumentar la concentración facilitando la adquisición de conocimientos, el desarrollo de habilidades y capacidades; crea un ambiente relajado y más participativo; activa la creatividad en cuanto que deben inventar, imaginar, descubrir, adivinar, esto con el fin de solucionar las diferentes situaciones. La creatividad, a su vez, estimula la actividad cerebral mejorando el rendimiento según los principios de la psicología del aprendizaje y crea una necesidad real de comunicación donde tienen la oportunidad de poner a prueba sus conocimientos y practicar tanto las destrezas de expresión como las de comprensión oral y escrita.

\section{Pruebas de efectos intersujetos}

Con respecto a los lugares no hubo diferencias estadísticamente significativas entre estos $(p=0,260)$, lo que demuestra que el aprendizaje en los tres corregimientos (Obonuco, Genoy y la Laguna) fue similar, tal como se muestra en la Figura 2, a diferencia de la evaluación de conocimientos previos (Ev1), en donde hubo diferencias en los tres lugares.

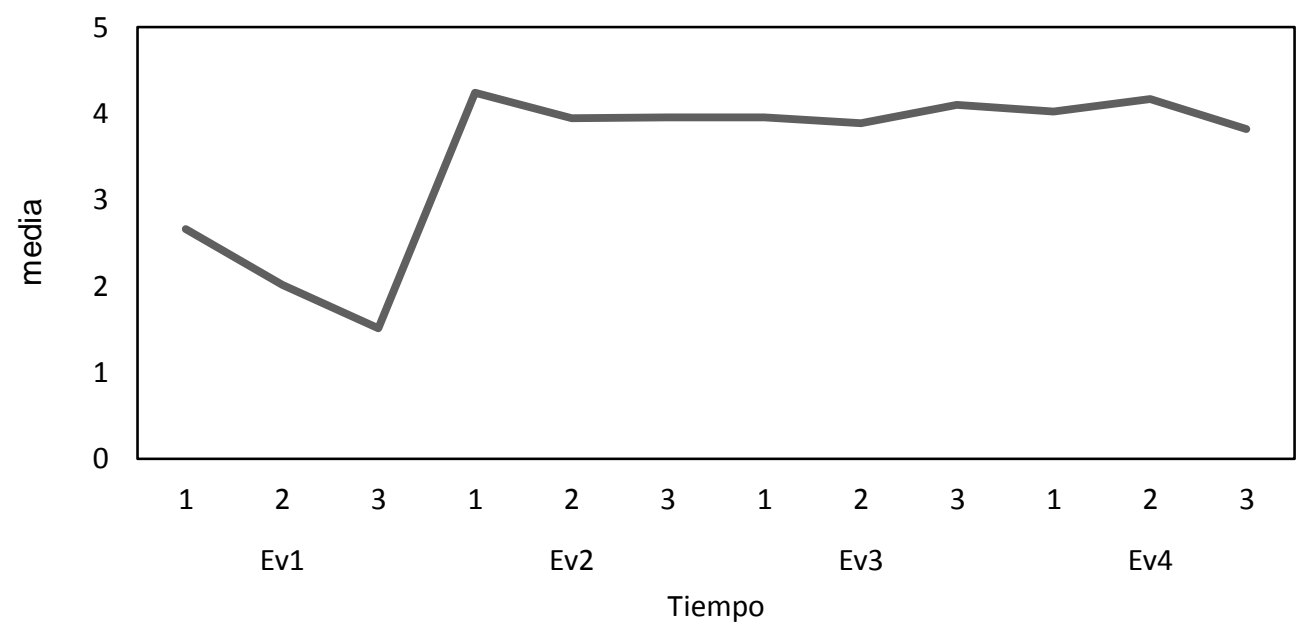

Fig. 2: Medias estimadas para test general en tiempo y lugar

Según las evaluaciones realizadas posteriormente al proceso de capacitación, en los tres corregimientos se obtuvo una media promedio de 4.01, lo que indica que la aplicación del material didáctico influyó de manera positiva en el aprendizaje al trascurrir el tiempo, según Beltrán (2017) la didáctica ayuda a que el aprendizaje sea permanente, donde el material didáctico logra que el adulto aprenda, recuerde y use esta información en su vida diaria al permitir que el ser humano reconozca los nuevos conocimientos, los revise, organice, restaure, asimile e interprete. Además, con la evaluación se logró evidenciar que el material es valioso por sus aportes en el aprendizaje de mujeres rurales; sin embargo, es susceptible de algunos ajustes para lograr un mayor impacto en el contexto local. En la Tabla 5 se presentan los diferentes aportes realizados a la fundamentación del material didáctico de seguridad alimentaria.

En este sentido, la opinión de las participantes respecto a cada uno de los juegos empleados en el desarrollo de las capacitaciones, contribuyó en la toma de decisiones para mejoras de forma y contenido: tamaño de imágenes y letra, colores, rediseño del juego "concéntrese en su salud" incorporando enfoque de género, aumento de productos y especies de cultivos andinos en los juegos "mercado familiar" y "planifica tu producción" respectivamente, y el cambio del tren alimenticio por canastas contenedoras de productos junto al plato de la familia colombiana planteado por el Instituto Colombiano de Bienestar Familiar- ICBF en el juego "sapo alimenticio". Además de ello, se evidenció la necesidad de incorporar conceptos de soberanía alimentaria desde el conocimiento ancestral en la diversidad de alimentos andinos, además los materiales deben ser cuidadosamente diseñados, atractivos, sencillos, con su propio control de error, deben progresar de lo sencillo a lo complejo y de lo concreto a lo abstracto.

\section{DISCUSIÓN FINAL}

La idiosincrasia de la mujer rural en la zona de investigación, tiende a valorar y respetar a sus pares, aspectos que generan satisfacción en la medida en que se logra compartir, por ello, los espacios de aprendizaje que les permiten dialogar e intercambiar experiencias, despiertan en cada participante gran interés por el análisis de problemas y situaciones que se evidencian con el desarrollo de cada uno de los juegos lo que conlleva a reflexionar acerca de su realidad, cuestionándose sobre su quehacer, asumiendo compromiso de cambio. 
Se evidencia que las temáticas tratadas con el uso del material didáctico de seguridad alimentaria persisten en el tiempo, posiblemente debido a que las mujeres rurales le dan mayor validez al proceso de formación que implica relaciones de confianza, de integración y participación, aspectos que se logra al emplear en las capacitaciones los distintos juegos que se destacan por ser contextualizados, sencillos y atractivos en su diseño lo que facilita la comprensión de contenidos, afianzando conocimientos útiles y prácticos, logrando mayor valoración y arraigo por su medio de vida.

Tabla 5: Aportes en la fundamentación del material didáctico de seguridad alimentaria

\begin{tabular}{lll} 
Módulo & Juego & Fundamentos de formación a resaltar con los juegos \\
\hline $\begin{array}{l}\text { La familia y su } \\
\text { compromiso } \\
\text { con la }\end{array}$ & $\begin{array}{l}\text { Rompecabezas "Rol } \\
\text { producción }\end{array}$ & $\begin{array}{l}\text { Los saberes ancestrales considerados como el conjunto de conocimientos, } \\
\text { prácticas, mitos y valores transmitidos de generación en generación; desde } \\
\text { el enfoque agrícola, los referentes a: siembra según calendario por fases } \\
\text { de la luna, manejo de cultivos considerando épocas de lluvia, abonado de } \\
\text { suelos y épocas de cosecha. }\end{array}$ \\
& $\begin{array}{l}\text { Principios agroecológicos para la toma de decisiones agricultura familiar en } \\
\text { pequeñas unidades de producción que rescatan razones culturales } \\
\text { heredadas como parte de su identidad y medios de vida. }\end{array}$
\end{tabular}

Disponibilidad y Lotería "Planifica Tu Los agroecosistemas tradicionales, especialmente aquellos en ambientes diversidad de Producción" marginales, poseen a menudo una estabilidad y una elasticidad importante, alimentos como resultado del alto nivel de diversidad estructural y funcional que se deriva del uso de policultivos, sistemas agroforestales y sistemas mixtos cultivos-animales.

Los requisitos básicos de un agroecosistema sustentable son la conservación de los recursos renovables, la adaptación del cultivo al ambiente y el mantenimiento de un nivel alto, pero estable, el fomento de la producción local de alimentos, adaptados al entorno socioeconómico y natural.

\begin{tabular}{|c|c|c|}
\hline $\begin{array}{l}\text { Importancia de } \\
\text { la huerta en la } \\
\text { economía } \\
\text { familiar }\end{array}$ & $\begin{array}{l}\text { Compraventa } \\
\text { "Mercado Familiar" }\end{array}$ & $\begin{array}{l}\text { Diversas iniciativas locales de desarrollo, buscan facilitar a nivel micro las } \\
\text { actividades productivas locales; igualmente otras se han basado en la } \\
\text { organización de redes de interdependencia entre empresas y actividades } \\
\text { ligadas a los mercados; el impulso de la diferenciación y calidad de } \\
\text { productos y procesos productivos, así como en la identificación de la } \\
\text { demanda y la emergencia de nuevas necesidades y mercados, aspectos a } \\
\text { considerar para fortalecer los ingresos de las familias productoras, siendo } \\
\text { de gran relevancia conservar la producción constante en la huerta } \\
\text { diversificada para garantizar la seguridad alimentaria y generar excedentes } \\
\text { de comercialización. }\end{array}$ \\
\hline
\end{tabular}

$\begin{array}{ll}\begin{array}{l}\text { Promoción de la } \\ \text { salud }\end{array} & \text { Concéntrese } \\ & \text { Salud" }\end{array}$

Propender por la inocuidad de los alimentos es una prioridad en la familia, ésta hace referencia al conjunto de características de los alimentos que garantizan que sean aptos para el consumo humano, y exigen el cumplimiento de una serie de condiciones y medidas necesarias durante la cadena agroalimentaria hasta el consumo y el aprovechamiento de los mismos, asegurando que una vez ingeridos no representen un riesgo que menoscabe la salud.

$\begin{array}{ll}\text { Alimentación } & \text { Sapo } \\ \text { saludable } & \text { "Sapo Alimenticio" }\end{array}$

La nutrición adecuada es un factor fundamental para el vigor y el desempeño de una persona, y para que funcione en su nivel óptimo debe consumir las cantidades adecuadas de alimentos que contengan los nutrientes esenciales para la vida humana.

El Plato saludable de la Familia Colombiana orienta a elegir una alimentación variada. Indica que, en cada tiempo de comida, se deben incluir alimentos de todos los grupos o su mayoría, en cantidades o porciones adecuadas.

\section{CONCLUSIONES}

En las tres zonas de estudio se presentó similar resultado en cuanto a la comprensión de los contenidos sobre seguridad alimentaria, evidenciando que el material didáctico es un recurso que facilita la comunicación y contribuye significativamente en la apropiación social del conocimiento.

La evaluación asertiva de juegos sobre distintos temas para la capacitación de adultos, representa un gran aporte para los profesionales y técnicos quienes pueden contar con materiales útiles y prácticos en su función de extensionistas rurales. Además, los ajustes planteados de forma participativa permitieron generar un material didáctico más contextualizado al territorio, permitiendo ser una representación de condiciones sociales, economías, culturales y ambientales de la comunidad. 


\section{REFERENCIAS}

Acosta, L., La relación entre los estilos de aprendizaje y el uso de las tecnologías de información y de comunicación en educación de personas adultas, Rev. Electrónica educare, 20(3), 1-18 (2016).

Aguilar, J. La evaluación educativa. México: Asociación Oaxaqueña de Psicología. (2011)

Alario-Hoyos, C., Estévez-Ayres, I y otros tres autores, Understanding Learners' Motivation and Learning Strategies in MOOCs. InternationalReview of Research in Open and Distributed Learning. 18(3), 119 - 137 (2017).

Beltrán, E.J., Los recursos didácticos y el aprendizaje significativo, Rev. Multidisciplinaria de investigación, 2(1), 33-38 (2017).

Cadena, A. Propuesta participativa de turismo rural sustentable, en la vereda San Felipe, quebrada Mijitayo, cuenca alta del río Pasto. Trabajo de grado para el título de ingeniero agroforestal, Universidad de Nariño, Pasto, Colombia. (2013).

Caniglia, G., John, B y otros seis utores "An experience-based learning framework", https://doi.org/10.1108/lJSHE-042015-0065, International Journal of Sustainability in Higher Education, 17(6), 827-852 (2016).

Carr, G.,Loucks, D y Bloschl, G. Gaining insight into interdisciplinary research and education programmes: Aframework for evaluation. Research Policy. 47(1). 35 - 48 (2018).

Chacón, P., La andragogía como disciplina propulsora de conocimiento en la educación superior. Revista Electrónica Educare, 16(1), 15-26. (2012).

De Backer, L., Van Keer, H., y Valcke, M., Exploring evolutions in reciprocal peer tutoring groups' socially shared metacognitive regulation and identifying its metacognitive correlates https://doi. org/10.1016/j.learninstruc.2015.04.001. Learning and Instruction, 38, 63-78, (2015).

De Souza, R., Portas, G., Ribeiro, F y Mamede-Neves, M, Design of didactic material for teaching and learning situations: the case of são tomé and príncipe, Africa. Problems of education, 74(1), 16 - 30 (2016).

Dunn, R., Dunn, K y Price,G,. Manual: inventario sobre los estilos de Enseñanza. Primera edición. 338 (1979).

Falcao, E., Moraes, M y Rossato, J. Evaluation of Virtual Objects: Contributions for the Learning Process. International Review of Research in Open and Distributed Learning. 17(6), 62 - 74 (2016).

Johnson D. W y Johnson R.T., Cooperative learning lesson structures. Edina, M.N,: Interaccion books. (1991).

Leberman, S., Mcdonald, L y Doyle, S., The Transfer of Learning. Participants' Perspectives of Adult Education and Training. Primera edicion. Gower Publishing. USA (2016).

López, M., y Malagón, G., Creating materials with ICT for CLIL lessons: A didactic proposal. Procedia - Social and Behavioral Sciences. 237(1), 633 - 637. (2017).

Lu Gram, J., Naomi, S, Bhim, D, Costello, A y Skordis-Worrall, J., Do Participatory Learning and Action Women's Groups Alone or Combined with Cash or Food Transfers Expand Women's Agency in Rural Nepal?, 10.1080/00220388.2018.1448069. The Journal of Development Studies, 55(8), 1670-1686 (2019).

Meij, H., Meij, J y Harmsen, R. Animated pedagogical agents effects on enhancing student motivation and learning in a science inquiry learning environment, 10.1007/s11423-015-9378-5, Education Tech Research Dev, 63(1), 381-403, (2015).

Moore, K., y Shemberger, M., Mass Communication Andragogy for Teaching Online Adult Learners, Teaching Journalism \& Mass Communication. 9(1): 35-40 (2019).

Mora, A., La evaluación educativa: Concepto, períodos y modelos. Rev. Electrónica Actualidades Investigativas en Educación, 4(2),1-26, (2004).

Muñoz, A.G. y Tejedor, F.J, Student perception of the value of ICT's in their learning strategies and their relation to performance, 10.5944/educXX1.19035, Educación XX1, 20(2), 137-159 (2017).

Narváez-Romo, Y., Luna, G., Leonel, H y Ruiz, J. Evaluación del Software Educativo Mundo Agroforestal con Jóvenes Rurales de Nariño, Colombia, 10.4067/S0718-07642017000200015. Información Tecnológica, 28(2), 135-140 (2017).

Nowak, M., Education as Support for the Integral Development of the Pupil, http://dx.doi.org/10.12775/PCh.2018.013, Paedagogia Christiana, 2(42), 11 - 32. (2018.)

Rajaee, S., Effects of e-learning on students 'motivation. Procedia- Social and Behavioral Sciences. 181(1) $423-430$. (2015).

Sánchez, O.R, Collazo, C.A y Jiménez, J.A., El trabajo colaborativo como estrategia didáctica para la enseñanza/ aprendizaje de la programación: una revisión sistemática de literatura, Rev. Tecnológicas, 21(41), 115-134, (2018).

Silva, A., Machado, A y Spanhol, F. Didactic Material Production Management. US-China Education Review. 6(2), 102 109 (2016).

Tipantuña, J., Uso de narrativas digitales como recurso didáctico para el aprendizaje de adultos: propuesta de diseño para su integración en el aprendizaje permanente. Revista Digital Publiseher. 4 (4), 29-40 (2019). 
Vasilaky, $\mathrm{K}$ y Islam, A. Competition or cooperation? Using team and tournament incentives for learning among female farmers in rural Uganda. World Development. 103(1), 216-225 (2018).

Walsh, G., Mcmillan, D y Mcguinnes, C, Playful, teaching and learning. Primera edición. Jude Bowen, (2017).

Yaqueno-Ortega, A.R., Luna-Cabrera, G.C. y Leonel, H. Eficacia del material didáctico 'agroforestería’ para agricultores de tres zonas del departamento de Nariño, 10.17151/luaz.2019.47.2. Rev. Luna Azul, 47, 21-35 (2018).

Zagarra, G.J, Carrillo, A.R y otros tres autores. El aprendizaje lúdico en el nivel medio superior, Rev. Divulgación científica,2(1), 861-873 (2016). 